\title{
Médiévales
}

Langues, Textes, Histoire

61 | automne 2011

La chair des émotions

\section{Prêcher les émotions incarnées. Évêques, mendiants et leurs publics dans l'Antiquité tardive}

Preaching embodied Emotions. Bishops, Beggars and their Audience in Late

Antiquity

Dionysios Stathakopoulos

\section{OpenEdition}

Journals

Édition électronique

URL : https://journals.openedition.org/medievales/6250

DOI : 10.4000/medievales. 6250

ISSN : $1777-5892$

Éditeur

Presses universitaires de Vincennes

Édition imprimée

Date de publication : 20 décembre 2011

Pagination : 25-37

ISBN : 978-2-84292-337-2

ISSN : 0751-2708

\section{Référence électronique}

Dionysios Stathakopoulos, «Prêcher les émotions incarnées. Évêques, mendiants et leurs publics dans l'Antiquité tardive », Médiévales [En ligne], 61 | automne 2011, mis en ligne le 17 janvier 2012, consulté le 22 avril 2022. URL : http://journals.openedition.org/medievales/6250 ; DOI : https:// doi.org/10.4000/medievales.6250 
Médiévales 61, automne 2011, p. 25-38

Dionysios STATHAKOPOULOS

\section{PRÊCHER LES ÉMOTIONS INCARNÉES. ÉVÊQUES, MENDIANTS ET LEURS PUBLICS DANS L'ANTIQUITÉ TARDIVE}

Je commencerai in medias res, avec le corps fugace des pauvres ${ }^{1}$. Il n'existe aucun texte prémoderne dont ils soient les auteurs, et leur parole apparaît comme un acte de ventriloquie produit par des personnes plus éduquées et occupant une position sociale plus élevée. Nous ne pouvons observer ou considérer leur corps et leurs émotions qu'à travers le langage, les perceptions et la rhétorique d'autrui - et cet obstacle ne peut guère être levé. Dans ce qui suit, je tenterai de conduire une lecture attentive d'un ensemble de textes spécifiques écrits à la fin du IV ${ }^{\mathrm{e}}$ siècle dans le but de revisiter de façon critique la connaissance que nous avons de certains groupes d'individus nécessiteux, et de soumettre nos sources à une analyse centrée sur le corps et les émotions. Que savons-nous du corps des pauvres? Comment était-il utilisé voire manipulé, dans quel but et avec quel effet - tant par leurs «propriétaires » que par ceux qui les décrivaient? Comment les descriptions rendent-elles compte de la mise en scène du corps des pauvres? Quelles émotions peut-on percevoir comme incorporées par les pauvres, et quelles réactions émotionnelles ont-ils provoquées chez ceux dont ils sollicitaient l'aumône?

En posant d'emblée toutes ces questions, je suis bien conscient que mon approche vise à traiter ensemble des émotions appartenant à différentes personnes et catégories sociales: en premier lieu, les émotions des prédicateurs, que l'on doit pour cette étude considérer comme identiques à celles exprimées dans leurs textes même si ce n'est en aucun cas certain; ensuite, les émotions que les sermons

1. Je tiens à remercier ici Barbara Rosenwein pour ses commentaires astucieux et Piroska Nagy pour sa traduction, ses questions et ses remarques, qui m'ont grandement aidé à améliorer ce texte. 
avaient pour but de provoquer. Enfin il sera question des émotions éprouvées par les mendiants lépreux eux-mêmes, sur lesquelles nous n'avons aucune autre information que ces sermons, rédigés bien loin d'eux. Nécessairement, compte tenu de la difficulté d'accès de l'historien à l'émotion d'autrefois, et plus particulièrement en raison des difficultés à parler par procuration - comme le faisaient d'ailleurs les auteurs des sources anciennes eux-mêmes - des émotions des «classes subalternes», notre proposition peut paraître fragile. Pourtant, nous saisissons probablement là une des rares possibilités de traiter le sujet. Cette entreprise tentera ainsi de retracer un mécanisme émotionnel mis en scène par les prédicateurs dans des sermons dont l'objectif est de resserrer les liens sociaux à travers la pratique de la charité dans la communauté chrétienne.

«Tout d'un coup, [les pauvres] sont partout ${ }^{2}$.» Les historiens ont proposé différentes théories pour expliquer le moment et les modalités de ce changement. Selon Évelyne Patlagean, l'augmentation de la population à partir des dernières décennies du Iv ${ }^{\mathrm{e}}$ siècle est marquée par une vague massive d'immigration dans les cités de la partie orientale de l'Empire, dont les gens vivant dans des états variés de pauvreté composaient une part importante ${ }^{3}$ : ils auraient incarné les perdants de l'essor démographique et économique de la Méditerranée orientale dans l'Antiquité tardive. Peter Brown propose une interprétation alternative du phénomène: selon lui, ce qui a rendu les pauvres «visibles» n'était pas seulement leur nombre. Il affirme audacieusement: «dans un sens, ce sont les évêques chrétiens qui ont inventé les pauvres ${ }^{4}$. En focalisant leur attention sur un groupe qui, jusqu'alors, était resté aux marges des documents écrits, les évêques y ont vu une manière d'encourager les dons. De cette façon, ils ont arrimé l'essor de l'Église au destin des pauvres par l'entremise de la charité ${ }^{5}$. Or l'identité de ces pauvres est mouvante: on y rencontre aussi bien des laboureurs migrants cherchant du travail, des veuves et des orphelins, des vieux, des malades, des invalides, enfin des lépreux, bannis de la société sans espoir d'emploi. C'est ce dernier groupe que j'ai choisi d'étudier.

Le corpus le plus riche et détaillé concernant les pauvres, les mendiants et les indigents, y compris les lépreux, provient des homélies des évêques du IV siècle finissant, notamment de Basile de Césarée, Grégoire de Nysse, Grégoire de Nazianze et Jean Chrysostome, tous appartenant au groupe à qui P. Brown a

2. P. Brown, Poverty and Leadership in the Later Roman Empire, Hanovre, New Haven et Londres, 2002, p. 11.

3. É. Patlagean, Pauvreté économique et pauvreté sociale à Byzance, IVe-VII siècles, Paris-La Haye, 1977, p. 426-429 (Civilisations et Sociétés, 48).

4. P. Brown, Poverty and Leadership..., p. 8.

5. Sur ce développement, le livre cité de P. Brown offre probablement la lecture la plus originale et astucieuse. 
attribué l'invention des pauvres. Les nécessiteux apparaissent comme un sujet de préoccupation majeur pour ces hommes d'Église: leurs écrits multiplient les allusions aux pauvres, incluant parfois des descriptions assez détaillées dans un effort constant d'émouvoir les fidèles, et d'inciter ces derniers à la compassion et à l'aumône. Pour mener l'étude, j'examinerai leurs homélies, des sermons pastoraux prêchés à leurs communautés, souvent retranscrits par des sténographes (ce qui n'empêche pas que les textes aient été retravaillés, en tout ou partie, pour aboutir à leur forme actuelle) ${ }^{6}$. Tous les auteurs cités étaient issus de milieux aisés, ils étaient bien formés à la rhétorique et donc, comme on peut l'imaginer, familiers d'une longue tradition de construction textuelle et de manipulation du public. Ces éléments doivent être pris en considération au regard de l'objectif de ces textes et de l'auditoire visé. La présentation des nécessiteux sert ainsi plusieurs fins. En tout premier lieu, il s'agissait d'émouvoir l'assemblée et de l'inciter à des actes charitables - aumône, hospitalité - ou du moins de lui faire éprouver de la sympathie pour les pauvres. À un niveau plus profond, symbolique et social, les homélies cherchaient à combler le fossé entre les nantis et les démunis, étant donné que ce fut, semble-t-il, plus difficile pour les chrétiens de l'élite que pour les non-chrétiens aisés de transcender leur statut social pour se rapprocher de leurs frères pauvres ${ }^{7}$. Le moyen utilisé par les évêques consistait à construire une proximité, une familiarité fictive avec les indigents, en soulignant l'identification des pauvres avec le Christ selon une idée centrale du chapitre 25 de l'Évangile selon Matthieu, et en suggérant qu'ils étaient des «prochains» attitrés et des favoris du Christ ${ }^{8}$.

Afin de poser les bases de la discussion, je présenterai pour commencer quelques passages clés de ces auteurs, provenant de trois homélies Sur l'amour des pauvres des deux Grégoire. Il est en général admis que les textes de Grégoire de Nysse ont été écrits après ceux de Grégoire de Nazianze, mais on peut les considérer ensemble avec profit vu qu'ils portent sur des sujets similaires. Nous n'avons pas de certitude quant aux dates où les homélies ont été prononcées, entre la fin des années 360 et le début des années $380^{9}$; pour mon propos, ce cadre temporaire provisoire doit suffire. J'y ajouterai des données provenant des homélies de Jean Chrysostome, prêtre et prédicateur à Antioche depuis 386 et archevêque de Constantinople de 398 à 404.

6. M. Cunningham, «Homilies», dans E. Jefrereys, R. Cormack et J. Haldon éd., Oxford Handbook of Byzantine Studies, Oxford, 2008, p. 872-881.

7. I. SANDwell, Religious Identity in Late Antiquity: Greeks, Jews and Christians in Antioch, Cambridge, 2007, p. 140, 205-6 et surtout B. LeYerLE, «John Chrysostom on Almsgiving and the Use of Money», The Harvard Theological Review, 47 (1994), p. 29-47.

8. S. R. Holman, The Hungry are dying : Beggars and Bishops in Roman Cappadocia, Oxford, 2001, p. 148.

9. Ibid., p. 144-148. 
Nous allons traiter de groupes de mendiants défigurés et mutilés qui souffrent de la lèpre. Il serait trop simple d'identifier cette dernière à la lèpre de l'époque moderne; nous devons accepter que, dans la société du IV siècle, un grand nombre de maladies de peau handicapantes ainsi que les séquelles d'infections gangréneuses mal guéries étaient regroupées sous la catégorie de la lèpre ${ }^{10}$. Voici la description de Grégoire de Nysse:

Vous voyez ces hommes : l'affreuse maladie les a métamorphosés en bêtes; au lieu d'ongles, elle leur a mis des morceaux de bois aux mains et aux pieds. Étranges empreintes laissées sur nos routes! Qui reconnaîtrait là un pas humain? Ces gens qui hier se tenaient droit, et regardaient vers le ciel, les voici aujourd'hui recourbés à terre, marchant à quatre pattes, quasiment changés en bêtes : écoutez le souffle rauque qui sort de leur poitrine; c'est ainsi qu'ils respirent. [...] Chez ces hommes, tout se passe comme s'ils changeaient de nature, perdaient les traits de leur espèce pour se muer en monstres. Leurs mains leur servent de pieds. Leurs genoux deviennent talons, leurs chevilles et leurs orteils, s'ils n'ont été complètement rongés, ils les traînent misérablement comme les chaloupes qui tirent les navires ${ }^{11}$.

Les corps défigurés des lépreux, d'apparence animale ${ }^{12}$, sont décrits en détail par les deux auteurs, ce qui suggère à la fois la présence de ces mendiants dans leur entourage, et le fait que ces derniers exhibaient leurs membres malades lors de la quête d'aumônes. De fait, les deux Grégoire et Jean Chrysostome décrivent ces mendiants apparaissant en groupes, se livrant à des danses macabres et à d'autres tours, exhibant leurs membres pourrissants ou leurs moignons, leurs ulcères cutanés ou leur visage aux yeux dévorés par la maladie, tout en chantant des lamentations ou des chants funèbres d'une voix incompréhensible afin d'amener le public urbain à la charité ${ }^{13}$. Ils chantaient leur abandon par leur famille, leur exclusion de la société, l'interdiction qui leur était faite de boire l'eau potable à la fontaine publique ou à la rivière ${ }^{14}$. Car il ne faut pas oublier que tout ceci se

10. S. R. Holman, «Constructed and Consumed: Everyday Life of the Poor in 4th century Cappadocia», dans W. Bowden, A. Gutteridge et C. Machado éd., Social and Political Life in Late Antiquity, Leyde-Boston, 2006, p. 452.

11. GrÉGoIRe DE Nysse, De pauperibus amandis, II (= In illud: Quatenus uni ex his fecistis mihi fecistis), A. VAN HeCK éd., Gregorii Nysseni opera, vol. 9.1, Leyde, 1967, p. 114-15; Traduction: F. QuÉRÉ, dans Riches et pauvres dans l'Église ancienne, textes présentés par A.-G. HAMmAN, Paris, 1982, p. 150-151. Ma présentation de la construction rhétorique de la lèpre suit le compte rendu excellent et pionnier de S. R. Holman, «Constructed and Consumed...», surtout le chap. 4.

12. Aretaeus, De curatione diuturnorum morborum libri duo, II 13, 1-3, K. Hude éd., Berlin, $1958^{2}$

13. Il est important de noter que pendant que les pauvres, et parmi eux les mendiants lépreux, peuplent les homélies de Jean Chrysostome, son professeur et contemporain, le grand sophiste Libanius, ne les mentionne jamais dans ses nombreux écrits.

14. GrÉGOIRE De NysSe, De pauperibus amandis, II..., p. 116-17. 
passait à une époque où les lépreux n'étaient pas encore pris en charge par des institutions charitables.

Ces textes peuvent nous aider à retracer les mécanismes émotionnels qui président à l'interaction entre les mendiants et leur public. Est-il nécessaire de rappeler que notre seul accès à ces échanges est fourni par les textes des prédicateurs, dont la construction fait partie du mécanisme émotionnel qu'il convient de retracer? La première émotion était souvent l'affliction : même les prédicateurs admettent qu'ils étaient dérangés par le spectacle des mendiants défigurés. Les émotions négatives (dégoût, horreur, répulsion et même haine) venaient ensuite rapidement ; les gens voulaient éviter les mendiants, détourner le regard et fuir: "Mais il n'est pas facile, dites-vous, de maîtriser ce hautle-cœur que les gens éprouvent instinctivement devant les malades. Ah! oui. Je suis de votre avis : ce n'est pas facile ${ }^{15}$ !» L'horreur qu'un tel corps éveillait était accrue par la vue inévitable de tout le groupe des malades. Parfois la réponse émotionnelle culminait en abus verbal voire physique ${ }^{16}$. Jean Chrysostome relate que le peuple contemplait les spectacles macabres des lépreux bouche bée et qu'il ressentait parfois de la Schadenfreude ${ }^{17}$, du plaisir procuré par la souffrance des autres - peut-être avec le soulagement de ne pas être à leur place et un sentiment de crainte devant la possibilité de succomber à la maladie. Parfois, à l'inverse, il ne manifestait aucune réponse émotionnelle, juste de l'insensibilité et de 1'indifférence ${ }^{18}$. Ces premiers contacts avec les textes nous amènent à penser que la vue du corps des mendiants était précisément l'aiguillon qui provoquait la réponse émotionnelle, engendrant ou non un geste social ou physique.

Les textes nous fournissent aussi un aperçu des émotions des mendiants eux-mêmes. D'après les sermons, ils ressentent de la pitié et de la haine pour euxmêmes ${ }^{19}$; ils sont les seuls à oser se regarder : «la nécessité leur fait surmonter l'horreur ${ }^{20}$ », mais comme ils «craignent de déshonorer la nature commune en en portant le titre», ils ressentent de la honte à être des humains :

Ils passent leur vie à gémir et les raisons de pleurer ne leur font jamais défaut. Il leur suffit de se voir pour réveiller leurs plaintes. Car ils ne savent s'ils doivent déplorer d'avoir perdu leurs membres plutôt que d'en conserver encore. Leur faut-il donc s'attrister que la nature les ait parfois épargnés plutôt qu'elle ne les ait mutilés? Ou regretter d'être les témoins de leur déchéance plutôt que de ne

15. Ibid., p. 125 ; traduction, p. 158.

16. GréGoire de NaZianZe, Discours XIV (De pauperum amore), 29, dans PG 35, col. 897A.

17. Jean Chrysostome, Discours sur 1 Cor. 21, 6, dans PG 61, col. 177.

18. Ibid., col 176-178; GrÉGoIRE de NAZIANZe, Discours XIV (De pauperum amore), 10, dans $P G$ 35, col. 869B.

19. GréGoire de Nazianze, op. cit.; GrÉGOIRE de Nysse, De pauperibus amandis, II..., p. 118.

20. Ibid., p. 119; traduction: p. 152. 
plus l'avoir sous les yeux lorsque la maladie les a rendus aveugles? Ou d'avoir de tels malheurs à raconter plutôt que d'en être les victimes muettes lorsque la lèpre a rongé leur langue? Ou de se nourrir misérablement d'une bouchée de pain plutôt que d'avoir perdu la bouche et de ne plus pouvoir s'alimenter comme tout le monde? Ou de sentir leur corps pourrir comme une charogne plutôt qu'être devenu complètement insensible? Où est leur vue? Leur odorat, leur toucher? Où sont ces autres sens que l'infection montante ronge peu à peu ${ }^{21}$ ?

Ce qui frappe particulièrement dans la reconstitution de ces rencontres, c'est l'importance qu'elles accordent aux émotions négatives. On s'attendrait au discours chrétien traditionnel, mettant l'accent sur la gratitude, la pitié et la sympathie. Or, si ces émotions ont bien évidemment leur place dans le discours des évêques, l'attention est certainement ailleurs.

La honte est un motif dominant dans les textes. Selon les homélies, les mendiants ont honte, alors même qu'ils sont souvent vus comme éhontés par les chrétiens en bonne santé. En effet, les attitudes reconnues comme des signes de la honte - baisser le visage et éviter le regard, tenir les épaules affaissées et prendre une posture voûtée en marchant les genoux pliés ${ }^{22}$ - sont notées par nos auteurs chez d'autres catégories de nécessiteux : Basile décrit des fermiers agenouillés et touchant leurs genoux pendant une disette à Césarée ${ }^{23}$, des pauvres silencieux rôdant ou étendus par terre ${ }^{24}$, en écho à Lazare qui, à la porte de l'homme riche dans le Nouveau Testament, pense à lui-même, selon Jean Chrysostome, comme à «une source de honte et de dérision ${ }^{25}$ ». Mais les mendiants lépreux semblent être au-delà de la possibilité même d'accomplir ces gestes. Endurcis par le dédain et les vexations dont ils étaient victimes, ils tentaient probablement d'inspirer un comportement charitable à leur égard soit d'une manière plus passive - en reconnaissant par là leur position dans la culture de la honte et de l'honneur soit, dans les cas évoqués ci-dessus, en sollicitant les aumônes avec des méthodes plus voyantes. Ainsi, leurs spectacles chantés et dansés - les aumônes devenant le prix payé pour le divertissement - pouvaient peut-être agir contre la répulsion qui empêchait l'aumône. Dans tous les cas, la méthode semble avoir fonctionné : il n'est fait aucun commentaire négatif sur ces manifestations et Jean Chrysostome

21. Ibid., p. 118; traduction: p. 153.

22. D. M. T. Fessler, «From Appeasement to Conformity: Evolutionary and Cultural Perspectives on Shame, Competition, and Cooperation », dans J. L. Tracy, R. W. Robins et J. Price TANGNEy éd., The Self-Conscious Emotions. Theory and Practice, New York-Londres, 2007, p. 174193 (ici p. 175).

23. Basile de CÉsARÉE, Discours VIII (Homilia dicta tempore famis et siccitatis), dans PG 31, col. 308AB.

24. GréGoIre de NysSe, De pauperibus amandis I (= De beneficentia), dans A. VAn HeCK éd., Gregorii Nysseni opera, vol. 9.1, Leyde, 1967, p. 97.

25. Jean Chrysostome, De Lazaro concio, I 7, 9, dans $P G$ 48, col. 971, 975. 
accuse même les spectateurs de ne donner que pour inciter les mendiants à recommencer leur spectacle ${ }^{26}$. Cela dit, la façon de mendier des lépreux nous paraît assez agressive - dans un contexte contemporain, on dirait qu'elle est «émotionnellement pauvre» (emotionally cheap) ${ }^{27}$-, et c'est avec difficulté que les prédicateurs tentaient de la réconcilier avec le modèle de Lazare, le mendiant biblique à la passivité absolue. Si tant est que nous puissions comparer nos propres expériences avec des pratiques aussi éloignées, nous pourrions nous demander (et projeter peut-être avec une infinie prudence nos réponses sur le passé) quelle attitude nous adopterions plus aisément: celle, passive, du mendiant qui s'assoit et attend ${ }^{28}$, ou celle du mendiant plus actif voire agressif? Dans nos sources, le fait que les récits relatant des pratiques agressives soient plus répandus semble signifier qu'en ce temps au moins ce genre d'attitudes était efficace pour attirer les aumônes.

Ce comportement provoquait aussi d'autres réactions bien différentes et reliées à la honte. Les mendiants étaient facilement accusés d'être paresseux et sans vergogne, tout particulièrement les hommes jeunes ${ }^{29}$. Cette observation n'était pas faite seulement par les évêques. Une loi de l'empereur Valentinien, promulguée le 20 juin $382^{30}$, décrète que tous les mendiants en bonne santé à Rome doivent être arrêtés et mis au travail par ceux qui les dénoncent à l'État, soit comme colons, soit comme esclaves, suivant leur statut d'homme libre ou non. Pourtant, selon Grégoire de Nazianze, les fidèles devaient aussi ressentir de la honte s'ils n'aidaient pas les mendiants:

Quelle honte pour nous, si après tous les bienfaits et toutes les promesses dont il nous comble, nous ne lui apportons pas ce seul présent: l'amour des autres! [...] Mes frères, ne soyons pas les mauvais économes des biens que l'on nous a confiés, si nous ne voulons pas entendre gronder la voix de Pierre: Rougissez, vous qui retenez le bien d'autrui ${ }^{31}$.

Et de continuer:

26. ID., Discours sur 1 Cor., 21, 6, dans PG 61, col. 177; voir aussi W. MAYER, «Poverty and Generosity Towards the Poor in the Time of John Chrysostom», dans S. R. Holman éd., Wealth and Poverty in Early Church and Society, Grand Rapids, 2008, p. 150-151.

27. B. F. Williams, «The Public I/Eye : Conducting Fieldwork to Do Homework on Homelessness and Begging in Two U.S. Cities», Current Anthropology, 35 (1995), p. $25-51$ (ici p. 26).

28. B. KeIFEnHEIM, «Körperinszenierung und Aneignung von sozialem Raum: Das Beispiel deutscher Obdachloser in Paris», Soziologus, 56 (2006), p. 173-192.

29. W. MAYER, «Poverty and Generosity...», p. 150.

30. CTh XIV 18,1 .

31. Grégoire de Nazianze, Discours XIV (De pauperum amore), 23-24, dans $P G 35$, col 888CD-889A; traduction: p. 121. 
C'est pourquoi, n'admirons pas toute espèce de santé et n'abominons pas toute maladie, n'attachons pas notre cœur à des richesses furtives plus qu'il n'est de mise, et ne courons pas après cette fumée où nous dissiperons une partie de notre âme. Ne nous défions pas de la pauvreté comme si elle était un sujet de mépris, de malédiction, de haine, mais sachons mépriser une santé stupide qui engendre le péché. Respectons la maladie qu'accompagne la sainteté et rendons hommage à ceux que leurs souffrances ont acheminés à la victoire ${ }^{32}$.

Les auditoires chrétiens du IV ${ }^{\mathrm{e}}$ siècle étaient convertis très récemment, de sorte qu'on peut supposer que le modèle de comportement qui leur était présenté par les prédicateurs, à savoir donner aux pauvres plutôt que se livrer à des actes d'évergétisme, était pour eux difficile à accepter ${ }^{33}$. Même l'Ancien Testament ne semble guère avoir d'égards pour les mendiants, si l'on considère les aphorismes de l'Ecclésiastique $(40,28-30)$ qui ont conservé leur popularité tout au long du Moyen Âge:

Mon fils, puisses-tu ne pas mener une vie de mendicité; mieux vaut mourir que de mendier. Quand un homme en est réduit à regarder vers la table d'un autre, sa vie ne saurait compter pour une vie. Car il souille son âme par des mets étrangers, ce dont se gardera l'homme instruit et bien élevé. Dans la bouche de l'homme sans pudeur la mendicité est douce; mais, dans ses entrailles, elle brûle comme un feu.

L'interaction de la honte avec l'infamie ou l'impudence, ou avec son antithèse, l'honneur, était bien sûr une composante cruciale de la culture gréco-romaine ${ }^{34}$. Toutefois les pauvres semblent en avoir été exclus : le " "sens de la honte" était un luxe que les pauvres ne pouvaient pas se permettre ${ }^{35} »$. Selon les mots de Télémaque dans L'Odyssée $(17,347)$ : «La honte n'est pas bonne à l'indigent. » Nous pouvons supposer sans trop de risque que les mendiants lépreux avaient jadis été des membres sains de la société, et qu'ils étaient donc bien conscients des normes sociales dominées par le couple de l'honneur et de la honte ${ }^{36}$. Si la honte est une émotion évaluée négativement par les autres, dans la mesure où elle témoigne d'une défaillance vis-à-vis des normes sociales et culturelles ${ }^{37}$, nous pouvons nous attendre à ce que les mendiants lépreux la ressentent, constamment

32. Ibid., col. 904B; traduction: p. 130 .

33. W. MAYER, «Poverty and Generosity...», p. 141.

34. D. L. Cairns, Aidos. The Psychology and Ethics of Honour and Shame in Ancient Greek Literature, Oxford, 1993 ; C. A. BARTON, Roman Honor : The Fire in the Bones, Berkeley-Los AngelesLondres, 2001.

35. R. A. KASTER, Emotion, Restraint, and Community in Ancient Rome, Oxford, 2005, p. 10.

36. Voir l'utile synthèse de P. Horden et N. Purcell, The Corrupting Sea. A Study of Mediterranean History, Oxford, 2000, p. 488-501.

37. W. Wong et J. TsaI, «Cultural Models of Shame and Guilt», dans J. L. TRACy, R. W. Robins et J. Price Tangney éd., The Self-Conscious Emotions. Theory and Practice, New York-Londres, 2007, p. 209-223 (ici p. 210). 
rappelés qu'ils étaient à leurs manquements. Qui plus est, leur honte aurait été exacerbée par la cause même de leur pauvreté et de leur indigence: la maladie. Les maladies de peau ont toujours été stigmatisantes, ce qui nous conduit à penser que les mendiants lépreux avaient deux raisons d'éprouver la honte: la pauvreté sociale et économique, et la maladie qui les défigurait.

Dans leur tentative pour construire une proximité sociale, créer une familiarité entre donateurs et mendiants, les prédicateurs étaient entravés par un grand nombre de facteurs. D'abord, la familiarité requiert une base commune pour qu'opère le rapprochement voire l'identification. Comment la trouver alors que les mendiants, à leurs propres yeux et aux yeux de bien des spectateurs, n'ont pas apparence humaine? Ensuite, comment les donateurs réussissaientils à dépasser le système dominant de l'honneur et de la honte pour assister des gens que toute leur éducation leur avait appris à considérer comme couverts de honte et répugnants? Il importe de noter que ces penseurs chrétiens ne cherchaient pas à dévaluer le système dominant de l'honneur et de la honte, mais plutôt à le reconfigurer, en plaçant les mendiants à l'intérieur d'un système d'où ils avaient été traditionnellement exclus. Dans la société méditerranéenne tardoantique, la honte était prioritairement assimilée à la peur du déshonneur, à la crainte de perdre la face devant les siens ${ }^{38}$. Or les mendiants n'étaient pas vus comme des égaux en termes sociaux, et pouvaient donc facilement être méprisés ou oubliés. Les prédicateurs en revanche, en tant que figures d'autorité et, dans ce cas précis, issus de la haute société aisée, pouvaient inspirer la crainte de la perte de l'honneur au sein de l'assemblée. Ils pouvaient susciter la peur de ne pas se conformer aux attentes qu'ils édictaient, ce qui signifiait se placer hors des demandes sociales chrétiennes nouvelles, et risquer le déshonneur pour cela.

Dans leur entreprise, les prédicateurs commencent donc par créer un espace de proximité. Assurément, les évêques incluent une dimension sensorielle dans la parenté fictive qu'ils élaborent entre mendiants et donateurs : ces derniers sont incités à regarder les corps défigurés, au lieu de détourner leur regard. À l'opposé, il y a cette autre façon de voir en restant bouche bée face aux lépreux et en éprouvant la Schadenfreude, ce «mieux vaut eux plutôt que moi»... C'est une idée qui se trouve parfois soutenue par la notion de théodicée:

C'est Dieu qui veut leur malheur, c'est Dieu qui fait notre prospérité. Qui suisje, moi, pour m'opposer à ses décrets, et me montrer meilleur que lui ? Que les maladies, les deuils, les privations les accablent, puisque Dieu l'a voulu ${ }^{39}$.

38. D. L. CAIRNS, op. cit., chap. 6, p. 343 sq.

39. GréGoire de NaZianZe, Discours XIV (De pauperum amore), 29, dans $P G 35$, col. 897AB; traduction: op. cit., p. 126. 
En outre, les fidèles étaient encouragés à toucher les lépreux, ce qui n'était pas chose aisée à demander à quiconque. Les gens exécraient les toucher, tant en raison des tabous bibliques (cf. Lévitique 13-14) que par peur de la contagion ${ }^{40}$. Arétée, médecin qui faisait autorité au sujet de la lèpre, avait affirmé catégoriquement que la maladie pouvait se propager facilement par la respiration ${ }^{41}$. Et pourtant, à peu près à la même époque, des femmes de l'élite romaine comme Fabiola ou l'impératrice Flacille étaient couvertes d'éloges parce qu'elles soignaient les malades de leurs propres mains ${ }^{42}$. Dans son raisonnement Grégoire de Nazianze écarte le danger de contagion et demande à l'assemblée de toucher les mendiants lépreux, de panser leurs plaies, de leur parler et de les questionner sur leur condition $^{43}$ : autrement dit, de les inclure dans la société humaine. Grégoire de Nysse utilise des comparaisons très fortes pour émouvoir l'auditoire:

Non, tu ne plains pas un être de ta race, son mal ne t'inspire qu'horreur, sa prière t'est odieuse et tu fuis son abord comme l'assaut d'un fauve. Réfléchis donc un peu: les anges, quoique purs esprits, n'hésitent pas à toucher l'homme, et ton corps de chair et de sang ne leur fait point d'horreur. Mais pourquoi évoquer les anges? Leur propre roi, le maitre de la béatitude céleste, s'est fait homme pour toi et s'est revêtu de cette chair immonde avec l'âme qui y est enfermée. Et cela, afin de guérir tes maux en te touchant de sa main. Mais toi qui partages la nature de ce malade, tu fuis celui qui est de ta race ${ }^{44}$ ?

Seule cette transgression, cette tentative de déplacer les frontières sociales et symboliques peuvent rendre possible une réponse émotionnelle positive face aux lépreux, et il semble que c'est bien ce que les évêques visaient en faisant honte à leur communauté ${ }^{45}$. En outre, toucher les lépreux doit apporter des bienfaits également aux fidèles en bonne santé, qui bénéficient ainsi d'une guérison spirituelle, souffrants qu'ils sont d'un manque d'humanité. Les mendiants lépreux deviennent alors les instruments mêmes de la rédemption incorporée:

Leurs mains mutilées savent encore prêter assistance, leurs pieds gangrenés peuvent toujours courir jusqu'à Dieu, leurs yeux se sont fondus, mais discernent

40. Ibid., 13, col. 873C.

41. Aretaeus, De curatione diuturnorum morborum libri duo, II, 13, 1 dans Aretaeus, éd. K. HudE, Berlin, s.d. (Corpus medicorum graecorum).

42. D. Stathakopoulos, «I Seek not my Own: Is there a Female Mode of Charity?», dans M. Gruenbart, M. Mulett et L. Theis éd., Female Founders in Byzantium and Beyond, Vienne, (à paraître).

43. Grégoire de NaZianze, Discours XIV, 27, col. 893B.

44. GRÉGOIRE DE NySSE, De pauperibus amandis II, p. 115 ; traduction: p. 151.

45. S. R. Holman, "Constructed and Consumed: Everyday Life of the Poor in 4th-century Cappadocia », dans W. Bowden, A. Gutteridge et C. Machado éd., Social and Political Life in Late Antiquity, Leyde-Boston, 2006, p. 441-464 (ici p. 453). 
pourtant les biens invisibles, à la lumière de l'âme. Ne méprise pas leurs corps monstrueux : quelque temps encore, et tu contempleras une vision plus étonnante que tout miracle. [...] Si nous tenons à être reçus par eux dans les tentes éternelles, ouvrons-leur dès maintenant nos portes. Si nous voulons guérir des plaies dont nous ont affligés nos péchés, guérissons d'abord les ulcères qui minent leur chair ${ }^{46}$.

Évidemment, il s'agit ici de la vision théologico-pastorale des prédicateurs, laquelle n'anéantit pas les hiérarchies sociales au sein desquelles vivent et agissent les donateurs, les évêques et les mendiants. À l'intérieur de cet espace précaire et étroit, les mendiants cherchent eux aussi à manipuler la honte: en se mettant en avant sur la scène publique urbaine, ils constituent un rappel désagréable de l'importance cardinale de la charité. Les passants peuvent bien détourner le regard, ils savent en même temps - entre autres parce que les prédicateurs leur rappellent constamment leurs devoirs envers les mendiants - qu'ils devraient leur faire l'aumône. En outre, on peut supposer que les mendiants eux-mêmes ont perçu un changement, aussi léger soit-il: par ces injonctions morales et religieuses constantes, les chrétiens étaient incités à les assister davantage que dans les sociétés païennes, parce que désormais ces actions étaient encadrées par l'Église ${ }^{47}$.

Au risque d'oublier les réalités auxquelles les mendiants lépreux devaient faire face, il importe de conclure en considérant rapidement ce qui se passait dans la vie de la cité, plutôt que dans les esprits et la rhétorique des prédicateurs. La fréquence des exhortations suggère que les attitudes ne changeaient qu'au prix des plus grandes difficultés. En outre, la lassitude de l'auditoire devait jouer un rôle. Jean Chrysostome insère dans son sermon un aparté sous la forme d'une apostrophe rhétorique émise par un membre de l'assemblée:

Depuis combien de temps vous ne cessez d'introduire continuellement des pauvres et des mendiants dans vos discours, et de nous prédire des malheurs, et de dénoncer la pauvreté à venir, n'ayant d'autre souci que de faire de nous des mendiants ${ }^{48}$ ?

Les émotions des lépreux eux-mêmes, les «vraies » émotions des prédicateurs et de leur public nous restent à jamais inconnues. La seule certitude dont nous disposons est fournie par les textes que je viens de parcourir: ils sont nombreux, explicites et détaillés, et ils suggèrent tous que les émotions représentées remplissaient une fonction pastorale importante auprès des évêques prédicateurs. Ces

46. GRÉGOIRE DE NYSSE, De pauperibus amandis II, op. cit., p. 123; traduction: p. 156-157; voir S. R. Holman, The Hungry..., p. 135, 144, 163.

47. A. PARkin, "You Do him no Service": an Exploration of Pagan Almsgiving», dans M. Atrins et R. Osborne éd., Poverty in the Roman World, Cambridge, 2006, p. 60-82 et P. Brown, Poverty and Leadership...

48. Jean Chrysostome, Discours sur 1 Cor., 30, 5, dans PG 61, col. 256. 
textes tiraient leurs preuves des observations qu'il était facile de corroborer et ainsi ils reflétaient, sinon la réalité, du moins une version de celle-ci que les prédicateurs et leur public étaient préparés à accepter comme vraie ou vraisemblable. Ils rendent compte des stratégies que les prédicateurs utilisaient pour manipuler leur auditoire, en jouant de l'empathie afin de transformer progressivement la répulsion en charité et en compassion. Leur emphase redoublée sur les émotions et sur le corps qui les incarne est un témoignage de la centralité et de l'efficacité, clairement perçues à l'époque, d'une telle approche.

Au début des années 370 l'institution révolutionnaire de Basile pour les pauvres et les démunis ouvrit ses portes, le Basilias, à l'extérieur de Césarée. Les lépreux faisaient partie des malades qui y étaient accueillis. D'après l'eulogie de Basile par Grégoire de Nazianze, l'affreux spectacle était désormais épargné aux citoyens, les lépreux venant trouver refuge au Basilias. Dans les décennies suivantes, les fondations d'institutions charitables de ce genre se sont multipliées, de sorte que ces «objets de haine» dérangeants parce que dépourvus d'humanité ont disparu de la place publique, leurs chants et spectacles ont cessé de hanter la cité $^{49}$. La preuve la plus vivante de leurs corps et de leurs émotions était désormais consignée sur le parchemin, dans les copies des sermons des prédicateurs.

Dionysios Stathakopoulos - Centre for Hellenic Studies \& Department of History, King's College London, Strand London WC2R 2LS, Royaume-Uni

49. F. Boulenger, Grégoire de Nazianze. Discours funèbres en l'honneur de son frère Césaire et de Basile de Césarée, Paris, 1908, ch. 63; A. CRislip, From Monastery to Hospital, Ann Arbor, 2005, p. 103-120 (surtout p. 113 sq.). 
Prêcher les émotions incarnées. Évêques, mendiants et leurs publics dans l'Antiquité tardive

Cette étude d'un corpus de sermons écrits par des évêques du $\mathrm{IV}^{\mathrm{e}}$ siècle de l'Orient méditerranéen porte sur les regards posés sur les mendiants souffrant de la lèpre. À cette époque, avant les fondations charitables, les lépreux étaient encore très visibles dans la cité. L'article explore les stratégies utilisées par les auteurs dans la description des mendiants lépreux, la manière dont ils attirent l'attention sur leurs corps mutilés, et incitent l'assemblée à la compassion et à la charité en construisant une proximité ou une relation fictive entre les mendiants et leur auditoire, et en identifiant les mendiants avec le Christ, ce qui permettait d'encourager les membres de l'assemblée à l'aumône dans la société chrétienne. Les émotions, surtout celles exprimées par le corps, sont centrales dans ces textes.

Lépreux - mendiants - charité - pauvreté - sermons.

Preaching embodied Emotions. Bishops, Beggars and their Audience in Late Antiquity

This is a study of a corpus of sermons by fourth-century bishops centred on beggars suffering from leprosy. The period in question was a time when such individuals were not yet institutionalized and as such were highly visible. The article explores the strategies employed by the authors in describing the leper beggars and focusing on their disfigured bodies in an effort to move their congregation to compassion and charity by constructing a fictive relatedness between the beggars and their audience as well as by stressing the identification of the beggars with Christ and thus their entitlement within a Christian society. Emotions, especially those expressed by the body, are central in these texts.

Beggars - lepers - charity - poverty - sermons. 
